\title{
Beziehungen zwischen Herz und Magen
}

Bei der Verfeinerung der modernen Herzdiagnostik fragt man sich mit Recht, ob die 1763 von Heberden als «Angina pectoris gastraler Genese», 1913 von Roemheld als «gastrocardialer Symptomkom-plex» und 1918 von Ceconí als «Pneumatosís gastríca mit Herzbeteili-gung» bezeichneten Krankheitserscheinungen heute noch einen ei-genen Platz in der klinischen Krankheitslehre beanspruchen dürfen.

Gastroenterologia, Vol. 81, No. 3 (1954) 12

178

Editorial

Der Arzt am Krankenbett ist es gewohnt, koordinierte Beschwerden von Herz und Magen häufig zu beobachten, wobei von alien Magen-beschwerden abgesehen sei, die sich durch ein dekompensiertes Herz oder in seinem Gefolge einfach erklären lassen. Läßt sich wirklich zeigen, daß Magenveränderungen als Ursache erheblicher Herzbe-schwerden verschiedenster Art auftreten können? Der sogenannte gastrophrenische Symptomenkomplex in seinen verschiedenen Modi-fikationen scheidet hier aus, da hierbei der Schmerz eindeutig auf das Zwerchfell zu beziehen ist.

Dagegen zeigten frühere Untersuchungen von Dietrich und Schwiegk u. a., daß bei der Hiatushernie des Ösophagus die Herzkranzgefäße im Zustand der eingeklemmten Hernie pathologisch durchblutet wer-den. Erst kürzlich haben Cassano, Tronchettí und Baschíeri (Rass. Fisiopat. 24, 1197-1329, 1952) in sehr gründlichen Untersuchungen Klinik und Genese der gastrocardialen Syndrome studiert. Dabei er-gab sich, daß fast immer während der Schmerzzustände gleichzeitig Veränderungen am Magen und Herzen bestanden. Beim Roemheld-schen Symptomkomplex, dessen Hauptcharakteristikum sie in einer pektanginösen Krise im Zusammenhang mit der Nahrungs-aufnahme und Aerophagie erblicken, beobachteten sie röntgeno-logisch einen stark luftgeblähten Magen und typische E KG-Veränderungen im Sinne einer Coronarinsuffizienz mit Senkung von S-T und Abflachung von T, während Magen und Herz außerhalb des Anfalls keine pathologischen Zeichen aufwiesen. Für den arrhythmischen Symptomkomplex nach Rosenbach, bei dem sich Aerophagie oder Aerocolie mit Beklemmungsgefühl infolge von Tachycardie oder Extrasystolie kombinieren, bestätigten sie ebenfalls die Koinzidenz beider Veränderungen im Anfall und die normalen Verhältnisse im Wohlbefinden. Der zirkulatorische Kollaps infolge Aerophagie, den sie mit dem alten «vertigo e stomacho laeso» identifizieren, sowie das cardiozirkulatorische Syndrom bei der «kleinen» nutritiven Allergie wird von ihnen ebenfalls herangezogen; auch hierbei fanden sie ähn-liche Abstimmungen der Untersuchungsergebnisse zueinander wie bei den anderen Krankheitserscheinungen. Untersuchungen des Herz-kymogramms, des Schlagvolumens, Minutenvolumens, der Blut-druckverhältnisse, die plethysmographisch und oscillographisch verfolgt wurden, sowie die Vektorcardiographie u. a. unterbauten ihre Befunde.

Es kann danach keinem Zweifel unterliegen, daß Veränderungen am Magen, insbesondere eine große Magenblase, aber auch allergisch 